PROCEEDINGS OF THE

AMERICAN MATHEMATICAL SOCIETY

Volume 139, Number 6, June 2011, Pages 2151-216

S 0002-9939(2010)10740-2

Article electronically published on November 29, 2010

\title{
OPTIMAL ESTIMATES FROM BELOW FOR BIHARMONIC GREEN FUNCTIONS
}

\author{
HANS-CHRISTOPH GRUNAU, FRÉDÉRIC ROBERT, AND GUIDO SWEERS
}

(Communicated by Walter Craig)

\begin{abstract}
Optimal pointwise estimates are derived for the biharmonic Green function under Dirichlet boundary conditions in arbitrary $C^{4, \gamma}$-smooth domains. Maximum principles do not exist for fourth order elliptic equations, and the Green function may change sign. The lack of a maximum principle prevents using a Harnack inequality as for second order problems and hence complicates the derivation of optimal estimates. The present estimate is obtained by an asymptotic analysis. The estimate shows that this Green function is positive near the singularity and that a possible negative part is small in the sense that it is bounded by the product of the squared distances to the boundary.
\end{abstract}

\section{INTRODUCTION}

It is well-known that the Green function $G(x, y)$ for second order elliptic equations on bounded domains can be estimated from above and from below by positive multiples of the same (positive) function, which is explicitly given in terms of the distances to the boundary, $d(x), d(y)$, and the distance $|x-y|$. See for example 3 . The behaviour of the biharmonic Green function for Dirichlet boundary conditions should be somehow similar but will have two crucial distinctions. The singularity of course is of lower order, namely $n-4$ instead of $n-2$ with $n$ the dimension, but a more serious distinction is the fact that the biharmonic Green function is not everywhere positive for most domains. Indeed, the few known domains with a biharmonic Green function of a fixed positive sign are balls in arbitrary dimensions, small perturbations of those balls and of some limaçons in 2 dimensions. See respectively [2, 11, 10] and [6, 7]. The results in [7] extend and correct [13].

It has been observed numerically on domains with a sign changing biharmonic Green function that the negative part is rather small and that it is also not located near the singularity. The aim of this paper is to give optimal estimates from below. Previous results concerning smallness of the negative part have been obtained in 10] for $n \geq 3$ and [4] for $n=2$. With the estimates for the absolute value of that Green function in [5], these previously known estimates are, when $n>4$, as follows:

$$
-c d(x)^{2} d(y)^{2} \leq G_{\Omega}(x, y) \leq c^{*}|x-y|^{4-n} \min \left(1, \frac{d(x)^{2} d(y)^{2}}{|x-y|^{4}}\right)
$$

Received by the editors June 11, 2010.

2010 Mathematics Subject Classification. Primary 35B51; Secondary 35J40, 35A08.

(C)2010 American Mathematical Society 
for all $x, y \in \Omega \subset \mathbb{R}^{n}$ and where $c, c^{*}$ are some positive constants only depending on the domain. The distance of $x$ to the boundary $\partial \Omega$ is defined by

$$
d(x):=\inf \left\{\left|x-x^{*}\right| ; x^{*} \in \partial \Omega\right\},
$$

and $G_{\Omega}$ denotes the said Green function. Let us remind the reader that the biharmonic Green function $G_{\Omega}$ is such that

$$
u(x)=\int_{\Omega} G_{\Omega}(x, y) f(y) d y
$$

solves

$$
\left\{\begin{array}{cc}
\Delta^{2} u=f & \text { in } \Omega, \\
u=|\nabla u|=0 & \text { on } \partial \Omega .
\end{array}\right.
$$

In (11) the dimension is restricted to $n>4$. As has been shown in 10 for $n=3,4$ and in 4 for $n=2$, the estimate from below in (1) holds in all dimensions. An estimate from above has also been proved for $n \leq 4$, but the formula for that estimate is different from (1). Those estimates can be found in [5] and contain the function $H_{\Omega}$ in (4).

The main result is the estimate from below in the following theorem. For the sake of completeness we include the estimate from above.

Theorem 1. Let $\Omega \subset \mathbb{R}^{n}(n \geq 2)$ be a bounded $C^{4, \gamma}$-smooth domain. Let $G_{\Omega}$ denote the biharmonic Green function in $\Omega$ for (2). Then there exist constants $c_{1} \geq 0, c_{2}>0$, depending on the domain $\Omega$, such that we have the following Green function estimate:

$$
c_{2}^{-1} H_{\Omega}(x, y) \leq G_{\Omega}(x, y)+c_{1} d(x)^{2} d(y)^{2} \leq c_{2} H_{\Omega}(x, y)
$$

for all $x, y \in \Omega$, where

$$
H_{\Omega}(x, y):= \begin{cases}|x-y|^{4-n} \min \left\{1, \frac{d(x)^{2} d(y)^{2}}{|x-y|^{4}}\right\} & \text { if } n>4, \\ \log \left(1+\frac{d(x)^{2} d(y)^{2}}{|x-y|^{4}}\right) & \text { if } n=4, \\ d(x)^{2-n / 2} d(y)^{2-n / 2} \min \left\{1, \frac{d(x)^{n / 2} d(y)^{n / 2}}{|x-y|^{n}}\right\} & \text { if } n=2,3 .\end{cases}
$$

The estimate from above follows from [5, 15, 16, so that only the estimate from below has to be proved here. One should observe that $G_{\Omega}(x, y)+c_{1} d(x)^{2} d(y)^{2} \geq 0$ was proved for some suitable $c_{1} \geq 0$ in [10]. The preceding result may be considered as an extension of the estimates in [10, showing that close to the pole the positive singular behaviour of the fundamental solution can also be seen in the Green function.

Due to the different behaviour of the Green function we need to distinguish between $n \geq 3$ and $n=2$ in proving Theorem 1 . Finally we should remark that the lack of a maximum principle not only results in sign changing Green functions but also complicates the proof of these estimates in the fourth order case. The proof in the second order case heavily depends on the Harnack inequality, which in turn depends on the maximum principle. 
An interesting consequence of Theorem 1 is a uniform local positivity result. When $n \geq 3$ this was proved in [10, while for $n=2$ we refer to [9, Theorem 6.15]. Here the emphasis is on the interplay between Theorem 1 and the following result. Moreover, we provide a proof for $n=2$ which is much simpler than the previous one and in the same spirit as the proof for $n \geq 3$.

Theorem 2. Let $\Omega \subset \mathbb{R}^{n}(n \geq 2)$ be a bounded $C^{4, \gamma}$-smooth domain. Let $G_{\Omega}$ denote the biharmonic Green function in $\Omega$ for (2). Then there exists a constant $r_{\Omega}>0$ such that

$$
\begin{gathered}
G_{\Omega}(x, y)>0 \text { for all } x, y \in \Omega \text { with }|x-y|<r_{\Omega} . \\
\text { 2. Some AUXILIARY RESULTS FOR } n \geq 3
\end{gathered}
$$

A careful inspection of the proofs in Nehari [17] and Grunau-Sweers [12] shows the following local estimate for the biharmonic Green function from below.

Proposition 3. Let $n \geq 3$. Then there exist constants $\delta_{n}>0$ and $c_{3}>0$, which depend only on the dimension $n$, such that the following holds true.

Assume $\Omega \subset \mathbb{R}^{n}$ to be a $C^{4, \gamma}$-smooth bounded domain and let $G_{\Omega}:=G_{\Delta^{2}, \Omega}$ denote the Green function for the biharmonic operator under Dirichlet boundary conditions. If

$$
|x-y| \leq \delta_{n} \max \{d(x), d(y)\}
$$

then we have

$$
G_{\Omega}(x, y)> \begin{cases}c_{3}|x-y|^{4-n} & \text { if } n>4, \\ c_{3} \log \left(1+\frac{1}{|x-y|^{4}}\right) & \text { if } n=4, \\ c_{3} d(x)^{1 / 2} d(y)^{1 / 2} & \text { if } n=3 .\end{cases}
$$

For the constant $\delta_{n}$, one may achieve that $\delta_{n} \geq 0.5$.

In dimension $n=2$ it seems impossible to achieve a global linear dependence of the radius of a ball of guaranteed positivity on the boundary distance; see Lemma 7 and [17]. In other words, the best result seems $\delta_{2}=\delta_{2}(\Omega)$, which strictly depends on $\Omega$.

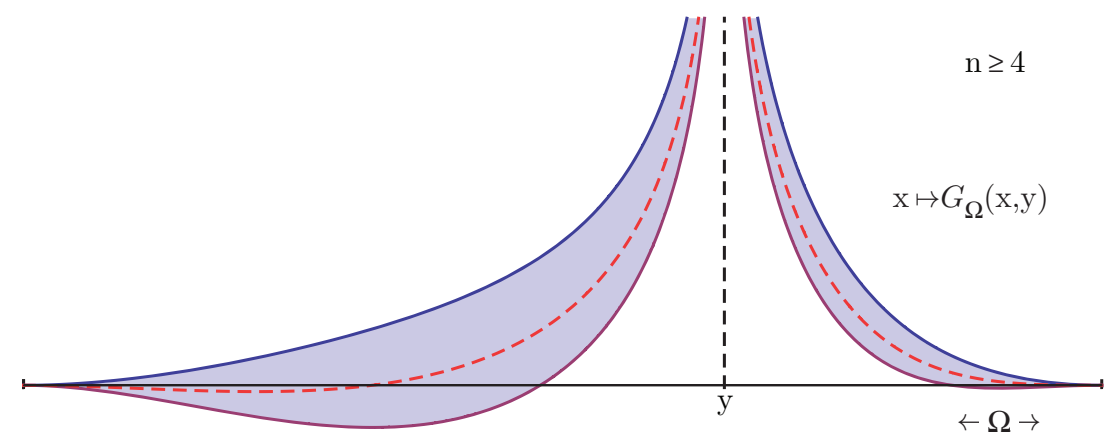

Figure 1. The dashed curve gives the typical behaviour of $G_{\Omega}$; the shaded area describes the band given by (3). 
Due to Proposition 3 and using the same constants $\delta_{n}>0$ as there, we may restrict ourselves in what follows to $x, y$ such that

$$
x, y \in \Omega, x \neq y,|x-y|>\delta_{n} \max \{d(x), d(y)\} .
$$

Lemma 4. Suppose that $n \geq 3$ and that $\Omega \subset \mathbb{R}^{n}$ is a bounded $C^{4, \gamma}$-smooth domain. Then for each $x_{0} \in \bar{\Omega}$ there exist a radius $r=r_{x_{0}}>0$ and a constant $C=C_{x_{0}}>0$ such that for all $x, y \in \Omega_{x_{0}, r}:=\bar{\Omega} \cap B_{r}\left(x_{0}\right)$ subject to condition (7) one has

$$
G_{\Omega}(x, y) \geq C|x-y|^{-n} d(x)^{2} d(y)^{2} .
$$

Proof. We only need to discuss $x_{0} \in \partial \Omega$ since for interior points $x_{0}$ one may choose $r=r_{x_{0}}>0$ so small that condition (7) becomes void.

We assume by contradiction that there exist $x_{k}, y_{k} \in \Omega_{x_{0}, 1 / k}=\bar{\Omega} \cap B_{1 / k}\left(x_{0}\right)$ subject to (7) such that

$$
G_{\Omega}\left(x_{k}, y_{k}\right)<\frac{1}{k}\left|x_{k}-y_{k}\right|^{-n} d\left(x_{k}\right)^{2} d\left(y_{k}\right)^{2} .
$$

In particular we have $x_{k} \rightarrow x_{0}, y_{k} \rightarrow x_{0}, d\left(x_{k}\right), d\left(y_{k}\right) \rightarrow 0,\left|x_{k}-y_{k}\right| \rightarrow 0$. Without loss of generality we may assume that $x_{0}=0$ and that the first unit vector $\vec{e}_{1}$ is the exterior unit normal to $\partial \Omega$ at $x_{0}$.

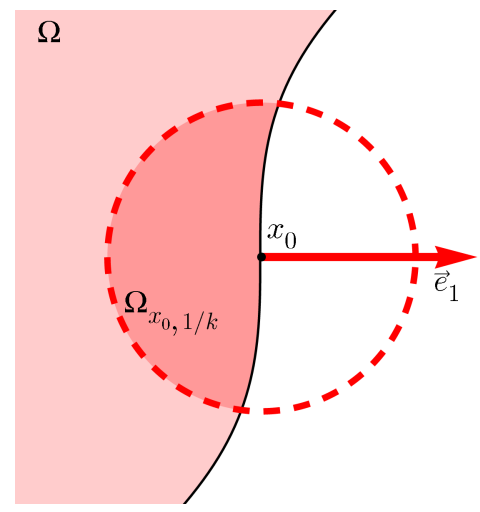

Figure $2 . \Omega$ and subdomain $\Omega_{x_{0}, 1 / k}$ for $x_{0} \in \partial \Omega$.

For $k$ large enough, we may define $\tilde{x}_{k} \in \partial \Omega$ as the closest boundary point to $x_{k}$. We introduce the rescaled biharmonic Green functions

$$
G_{k}(\xi, \eta):=\left|x_{k}-y_{k}\right|^{n-4} G_{\Omega}\left(\tilde{x}_{k}+\left|x_{k}-y_{k}\right| \xi, \tilde{x}_{k}+\left|x_{k}-y_{k}\right| \eta\right)
$$

for

$$
\xi, \eta \in \Omega_{k}:=\frac{1}{\left|x_{k}-y_{k}\right|}\left(-\tilde{x}_{k}+\Omega\right) .
$$

Since $\tilde{x}_{k} \rightarrow 0$, the exterior unit normal at $\partial \Omega$ converges to the first unit vector, and so we conclude that

$$
\Omega_{k} \rightarrow \mathcal{H}:=\left\{x: x_{1}<0\right\} \text { locally uniformly for } k \rightarrow \infty .
$$

It was proved in [10, Lemma 7] that locally uniformly in $\mathcal{H} \times \mathcal{H} \backslash\{(\xi, \xi) ; \xi \in \mathcal{H}\}$

$$
G_{k}(\xi, \eta) \rightarrow G_{\mathcal{H}}(\xi, \eta)=\frac{1}{4 n e_{n}}|\xi-\eta|^{4-n} \int_{1}^{\left|\xi^{*}-\eta\right| /|\xi-\eta|}\left(v^{2}-1\right) v^{1-n} d v
$$


where $\xi^{*}=\left(-\xi_{1}, \xi_{2}, \ldots, \xi_{n}\right)$ and $e_{n}$ is the $n$-dimensional volume of $B_{1}(0) \subset \mathbb{R}^{n}$. We remark that this step and in particular the required uniqueness proof for $G_{\mathcal{H}}$ were carried out in Grunau-Robert [10] using the assumption $n \geq 3$. The necessary modifications for $n=2$ are emphasized in the proofs of Lemmata 7 and 6 . Assumption (9) gives

$$
G_{k}\left(\xi_{k}, \eta_{k}\right)=\left|x_{k}-y_{k}\right|^{n-4} G_{\Omega}\left(x_{k}, y_{k}\right)<\frac{1}{k}\left|x_{k}-y_{k}\right|^{-4} d\left(x_{k}\right)^{2} d\left(y_{k}\right)^{2},
$$

where

$$
\begin{aligned}
\xi_{k} & =\frac{1}{\left|x_{k}-y_{k}\right|}\left(x_{k}-\tilde{x}_{k}\right), \quad \eta_{k}=\frac{1}{\left|x_{k}-y_{k}\right|}\left(y_{k}-\tilde{x}_{k}\right), \\
\left|\xi_{k}\right| & =\frac{d\left(x_{k}\right)}{\left|x_{k}-y_{k}\right|} \leq \frac{1}{\delta_{n}}, \quad\left|\xi_{k}-\eta_{k}\right|=1 .
\end{aligned}
$$

After passing to a further subsequence we find $\xi, \eta \in \overline{\mathcal{H}}$ with $\xi=\lim _{k \rightarrow \infty} \xi_{k}$, $\eta=\lim _{k \rightarrow \infty} \eta_{k}$. In view of the local smooth convergence of $G_{k}$ to the biharmonic Green function $G_{\mathcal{H}}$ in the half space $\mathcal{H}$, Boggio's formula and $|\xi-\eta|=1$, there exists a positive constant $\sigma>0$ such that for $k$ large enough,

$$
\begin{gathered}
G_{k}\left(\xi_{k}, \eta_{k}\right) \geq \sigma d\left(\xi_{k}\right)^{2} d\left(\eta_{k}\right)^{2}=\sigma\left(\frac{d\left(x_{k}\right)}{\left|x_{k}-y_{k}\right|}\right)^{2}\left(\frac{d\left(y_{k}\right)}{\left|x_{k}-y_{k}\right|}\right)^{2} \\
=\sigma\left|x_{k}-y_{k}\right|^{-4} d\left(x_{k}\right)^{2} d\left(y_{k}\right)^{2} .
\end{gathered}
$$

This contradicts (10), and the proof of the lemma is complete.

\section{Proof of the main estimate for $n \geq 3$}

Supposing that (6) holds, i.e. $|x-y| \leq \delta_{n} \max \{d(x), d(y)\}$, one finds that even $\left(1-\delta_{n}\right)|x-y| \leq \delta_{n} \min \{d(x), d(y)\}$, and hence

$$
\frac{d(x) d(y)}{|x-y|^{2}} \geq \frac{1-\delta_{n}}{\delta_{n}^{2}}
$$

Therefore, in that case the estimate from below in Theorem 1 follows directly from Proposition 3 . Hence, we may assume from now on, again, that $x, y$ are subject to condition (7). Applying a compactness argument to

$$
\bar{\Omega}=\bigcup_{x_{0} \in \bar{\Omega}} \Omega_{x_{0}, r_{x_{0}} / 2}
$$

we see that there exist positive numbers $r>0, c_{4}>0$, such that $|x-y| \leq r$ implies that $G_{\Omega}(x, y) \geq c_{4}|x-y|^{-n} d(x)^{2} d(y)^{2}$. If $|x-y| \geq r$, we take from [5] (cf. also [9]) that $G_{\Omega}(x, y) \geq-c_{5}|x-y|^{-n} d(x)^{2} d(y)^{2}$ so that

$$
G_{\Omega}(x, y)+2 c_{5}|x-y|^{-n} d(x)^{2} d(y)^{2} \geq c_{5}|x-y|^{-n} d(x)^{2} d(y)^{2} .
$$

Since $|x-y|^{-n} \leq r^{-n}$ we end up with

$$
G_{\Omega}(x, y)+c_{6} d(x)^{2} d(y)^{2} \geq c_{5}|x-y|^{-n} d(x)^{2} d(y)^{2}
$$

and positive constants $c_{5}, c_{6}>0$ in this case. The proof of Theorem 1 for $n \geq 3$ is complete. 


\section{Auxiliary Results FOR $n=2$}

Lemma 5. Let $\mathcal{H}=\left\{x \in \mathbb{R}^{2}: x_{1}<0\right\}$ and let $\tilde{G} \in C^{4}(\overline{\mathcal{H}} \times \overline{\mathcal{H}} \backslash\{(x, x): x \in \overline{\mathcal{H}}\})$ be a biharmonic Green function with Dirichlet boundary condition. That is,

$$
\int_{\mathcal{H}} \tilde{G}(x, .) \Delta^{2} \varphi d y=\varphi(x)+\int_{\partial \mathcal{H}}\left(\Delta \tilde{G}(x, .) \partial_{\nu} \varphi-\varphi \partial_{\nu} \Delta \tilde{G}(x, .)\right) d \sigma
$$

for all $\varphi \in C_{c}^{4}(\overline{\mathcal{H}})$ and $x \in \Omega$. Moreover, we assume that $\tilde{G}(x, y)=\tilde{G}(y, x)$ for all $x \neq y$ and that a growth condition holds at infinity:

$$
|\tilde{G}(x, y)| \leq C\left(1+|x|^{2}+|y|^{2}\right)\left(1+(\log |x|)_{+}+(\log |y|)_{+}\right) .
$$

Then $\tilde{G}$ is uniquely determined and given by Boggio's formula [2]:

$$
\tilde{G}(x, y)=G_{\mathcal{H}}(x, y)=\frac{1}{8 \pi}|x-y|^{2} \int_{1}^{\left|x^{*}-y\right| /|x-y|} \frac{v^{2}-1}{v} d v
$$

with $x^{*}=\left(-x_{1}, x_{2}\right)$.

Proof. We choose some arbitrary $x \in \mathcal{H}$ and keep it fixed in what follows. We write

$$
\tilde{G}(x, y)=G_{\mathcal{H}}(x, y)+H(x, y),
$$

where $H$ is a regular function in $\overline{\mathcal{H}} \times \overline{\mathcal{H}}$ and $\Delta_{y}^{2} H(x,.) \equiv 0$ in $\mathcal{H}$, and where $H(x, y)=\partial_{y_{1}} H(x, y)=0$ for $y_{1}=0$. According to [8, 14, or by checking directly, with $y^{*}=\left(-y_{1}, y_{2}\right)$,

$$
H^{*}(x, y):= \begin{cases}H(x, y) & \text { if } y_{1} \leq 0, \\ -H\left(x, y^{*}\right)-2 y_{1}\left(\frac{\partial H}{\partial y_{1}}\right)\left(x, y^{*}\right)-y_{1}^{2}\left(\Delta_{y} H\right)\left(x, y^{*}\right) & \text { if } y_{1}>0\end{cases}
$$

satisfies $H^{*}(x,.) \in C^{4}\left(\mathbb{R}^{2}\right)$ and is biharmonic on $\mathbb{R}^{2}$. Since $G_{\mathcal{H}}(x, y)$ satisfies (11), so does $H(x, y)$. So we have

$$
|H(x, y)| \leq C_{x}\left(1+|y|^{2}(\log |y|)_{+}\right) .
$$

Using local elliptic estimates and their scaling behaviour for biharmonic functions satisfying Dirichlet boundary conditions on $\partial \mathcal{H}$, that is,

we find that

$$
\left\|D^{\alpha} H(x, .)\right\|_{L^{\infty}\left(B_{R} \cap \mathcal{H}\right)} \leq \frac{C}{R^{|\alpha|}}\|H(x, .)\|_{L^{\infty}\left(B_{2 R} \cap \mathcal{H}\right)},
$$

$$
\left|\nabla_{y} H(x, y)\right| \leq C_{x}\left(1+|y|(\log |y|)_{+}\right), \quad\left|\nabla_{y}^{2} H(x, y)\right| \leq C_{x}\left(1+(\log |y|)_{+}\right) .
$$

Having these estimates for $H(x, y)$ and these first two derivatives, we have an estimate for $H^{*}(x, y)$ and may repeat the above arguments to find similar estimates for the derivatives of $H^{*}(x, y)$ and even

$$
\left|\nabla_{y}^{3} H^{*}(x, y)\right| \leq C_{x} \frac{\left(1+(\log |y|)_{+}\right)}{1+|y|} .
$$

The maximum principle applied to the harmonic function $\nabla_{y} \Delta_{y} H^{*}(x,$.$) shows that$

$$
\left\|\nabla_{y} \Delta_{y} H^{*}(x, .)\right\|_{C^{0}\left(\overline{B_{R}(0)}\right)} \leq C \frac{(1+|\log | R||)}{1+|R|} .
$$

Letting $R \rightarrow \infty$ yields

$$
\nabla_{y} \Delta_{y} H^{*}(x, .) \equiv 0, \quad \Delta_{y} H^{*}(x, .)=a(x)
$$


with a suitable function $a($.$) . This shows that any \nabla_{y}^{3} H^{*}(x,$.$) is harmonic and,$ as shown above, $\nabla_{y}^{3} H^{*}(x, y) \rightarrow 0$ as $y \rightarrow \infty$. Hence, any $\nabla_{y}^{3} H^{*}(x,.) \equiv 0$, and by Taylor's formula and observing the boundary data we conclude that

$$
H^{*}(x, y)=b(x) y_{1}^{2}
$$

with a suitable function $b($.$) . By symmetry H(x, y)=H(y, x)$, and so $H(x, y)=$ $b(y) x_{1}^{2}=b(x) y_{1}^{2}=b x_{1}^{2} y_{1}^{2}$, where $b \in \mathbb{R}$ is a suitable constant. Finally, the growth condition leads to $b=0, H(x,.) \equiv 0$, and $\tilde{G}=G_{\mathcal{H}}$.

Lemma 6 (Estimates near the boundary). Suppose that $n=2$ and that $\Omega \subset \mathbb{R}^{2}$ is a bounded $C^{4, \gamma}$-smooth domain. Then for each $x_{0} \in \partial \Omega$ there exist a radius $r=r_{x_{0}}>0$ and a constant $C=C_{x_{0}}>0$ such that for all $x, y \in \Omega_{x_{0}, r}:=\bar{\Omega} \cap B_{r}\left(x_{0}\right)$ one has

$$
G_{\Omega}(x, y) \geq C d(x) d(y) \min \left\{1, \frac{d(x) d(y)}{|x-y|^{2}}\right\} .
$$

Proof. We assume by contradiction that there exist $x_{k}, y_{k} \in \Omega_{x_{0}, 1 / k}=\bar{\Omega} \cap B_{1 / k}\left(x_{0}\right)$ such that

$$
G_{\Omega}\left(x_{k}, y_{k}\right)<\frac{1}{k} d\left(x_{k}\right) d\left(y_{k}\right) \min \left\{1, \frac{d\left(x_{k}\right) d\left(y_{k}\right)}{\left|x_{k}-y_{k}\right|^{2}}\right\} .
$$

In particular we have $x_{k} \rightarrow x_{0}, y_{k} \rightarrow x_{0}, d\left(x_{k}\right), d\left(y_{k}\right) \rightarrow 0,\left|x_{k}-y_{k}\right| \rightarrow 0$. Without loss of generality we may assume that $x_{0}=0$ and that the first unit vector $\vec{e}_{1}$ is the exterior unit normal to $\partial \Omega$ at $x_{0}$. After possibly passing to a subsequence, it is enough to consider one of the following two cases.

First case: $\left|x_{k}-y_{k}\right| \geq \frac{1}{2} \max \left\{d\left(x_{k}\right), d\left(y_{k}\right)\right\}$. This proof is the same as for Lemma 4 . only proving the required uniform bounds for $G_{k}$ is slightly more involved. The arguments are sketched below in the second case. Thanks to Lemma 5 the convergence proof of [10, Lemma 7] can be extended to $n=2$. One should observe that also the symmetry carries over to the limit.

Second case: $\left|x_{k}-y_{k}\right|<\frac{1}{2} \max \left\{d\left(x_{k}\right), d\left(y_{k}\right)\right\}$. Observe that in this case

$$
d\left(x_{k}\right)<2 d\left(y_{k}\right)<4 d\left(x_{k}\right)
$$

and

$$
\left|x_{k}-y_{k}\right|<\min \left\{d\left(x_{k}\right), d\left(y_{k}\right)\right\}
$$

The assumption gives the fact that

$$
G_{\Omega}\left(x_{k}, y_{k}\right)<\frac{1}{k} d\left(x_{k}\right) d\left(y_{k}\right)
$$

In this case we rescale differently; however, $\tilde{x}_{k} \in \partial \Omega$ again denotes the closest boundary point to $x_{k}$. We introduce the rescaled biharmonic Green functions

$$
G_{k}(\xi, \eta):=d\left(x_{k}\right)^{-2} G_{\Omega}\left(\tilde{x}_{k}+d\left(x_{k}\right) \xi, \tilde{x}_{k}+d\left(x_{k}\right) \eta\right)
$$

for

$$
\xi, \eta \in \Omega_{k}:=\frac{1}{d\left(x_{k}\right)}\left(-\tilde{x}_{k}+\Omega\right) .
$$

Since $\tilde{x}_{k} \rightarrow 0$, the exterior unit normal at $\partial \Omega$ converges to the first unit vector, and so we conclude that

$$
\Omega_{k} \rightarrow \mathcal{H}:=\left\{x: x_{1}<0\right\} \text { locally uniformly for } k \rightarrow \infty .
$$


For

$$
\xi_{k}=\frac{1}{d\left(x_{k}\right)}\left(x_{k}-\tilde{x_{k}}\right), \quad \eta_{k}=\frac{1}{d\left(x_{k}\right)}\left(y_{k}-\tilde{x_{k}}\right),
$$

the assumption (14) transforms into

$$
G_{k}\left(\xi_{k}, \eta_{k}\right)<\frac{1}{k} d_{k}\left(\xi_{k}\right) d_{k}\left(\eta_{k}\right)<\frac{2}{k}
$$

where $d_{k}:=d\left(., \partial \Omega_{k}\right)$. Since $\xi_{k}, \eta_{k}$ are bounded and their boundary distances are uniformly bounded from below by $1 / 2$, we find after passing to a further subsequence that $\xi_{k} \rightarrow \xi_{\infty} \in \mathcal{H}, \eta_{k} \rightarrow \eta_{\infty} \in \mathcal{H}$. We claim that we have local uniform convergence in $\mathcal{H} \times \mathcal{H}$ (including the diagonal) of $G_{k}$ to $G_{\mathcal{H}}$, since we are in dimension $n=2$. To see this we observe first that Krasovski1-type estimates (see [15, 16] and also [9, Theorem 4.20]) yield at a first instance useful information only for the third derivatives. We have

Making use of

$$
\left|\nabla_{\xi, \eta}^{3} G_{k}(\xi, \eta)\right| \leq \frac{C}{|\xi-\eta|} \text { uniformly in } k .
$$

$$
\forall \xi \in \partial \Omega_{k}, \eta \in \Omega_{k}: \quad \nabla_{\xi} \nabla_{\eta} G_{k}(\xi, \eta)=0, \quad \nabla_{\eta}^{2} G_{k}(\xi, \eta)=0
$$

and of

$$
\forall \xi \in \Omega_{k}, \eta \in \partial \Omega_{k}: \quad \nabla_{\xi} \nabla_{\eta} G_{k}(\xi, \eta)=0, \quad \nabla_{\xi}^{2} G_{k}(\xi, \eta)=0,
$$

we obtain upon integration that

$$
\left|\nabla_{\xi, \eta}^{2} G_{k}(\xi, \eta)\right| \leq C\left(1+(\log |\xi|)_{+}+(\log |\eta|)_{+}+|\log | \xi-\eta||\right) \text { uniformly in } k
$$

and further that

$$
\begin{gathered}
\left|\nabla_{\xi, \eta} G_{k}(\xi, \eta)\right| \leq C\left(1+(\log |\xi|)_{+}+(\log |\eta|)_{+}\right)(1+|\xi|+|\eta|) \text { uniformly in } k, \\
\left|G_{k}(\xi, \eta)\right| \leq C\left(1+(\log |\xi|)_{+}+(\log |\eta|)_{+}\right)\left(1+|\xi|^{2}+|\eta|^{2}\right) \text { uniformly in } k .
\end{gathered}
$$

Now, one may proceed further as in [10, Lemma 7]. So, (15) yields $G_{\mathcal{H}}\left(\xi_{\infty}, \eta_{\infty}\right) \leq 0$, while Boggio's formula shows that $G_{\mathcal{H}}(\xi, \eta)>0$ (even if $\xi=\eta$ ), since $\xi, \eta \in \mathcal{H}$ are interior points.

Lemma 7 (Estimates in the interior). Suppose that $n=2$ and that $\Omega \subset \mathbb{R}^{2}$ is a bounded $C^{4, \gamma}$-smooth domain. Then for each $x_{0} \in \Omega$ there exist a radius $r=r_{x_{0}}>0$ and a constant $C=C_{x_{0}}>0$ such that for all $x, y \in \Omega_{x_{0}, r}:=\bar{\Omega} \cap B_{r}\left(x_{0}\right)$ one has

$$
G_{\Omega}(x, y) \geq C d(x) d(y) \min \left\{1, \frac{d(x) d(y)}{|x-y|^{2}}\right\} .
$$

Proof. Since $x_{0} \in \Omega$, we have that $G_{\Omega}\left(x_{0}, x_{0}\right)>0$ (see [17, p. 115]). Since $G_{\Omega}$ is continuous, there exists $r, c>0$ such that $B_{r}\left(x_{0}\right) \Subset \Omega$ and $G_{\Omega}(x, y)>c$ for all $x, y \in B_{r}\left(x_{0}\right)$. This yields (16) since $\Omega$ is bounded.

\section{Proof of the main estimate for $n=2$}

Combining Lemmas 6 and 7 and applying a compactness argument to

$$
\bar{\Omega}=\bigcup_{x_{0} \in \bar{\Omega}} \Omega_{x_{0}, r_{x_{0}} / 2}
$$

we find: 
Corollary 8. Suppose that $n=2$ and that $\Omega \subset \mathbb{R}^{2}$ is a bounded $C^{4, \gamma}$-smooth domain. Then there exist a radius $r>0$ and a constant $C>0$ such that for all $x_{0} \in \bar{\Omega}$ and for all $x, y \in \Omega_{x_{0}, r}:=\bar{\Omega} \cap B_{r}\left(x_{0}\right)$ one has

$$
G_{\Omega}(x, y) \geq C d(x) d(y) \min \left\{1, \frac{d(x) d(y)}{|x-y|^{2}}\right\} .
$$

This step provides in particular a different and simpler proof for the local positivity statement from [9, Theorem 6.15] which was proved first by Dall'Acqua, Meister, and Sweers [4.

Proof of Theorem 1. If $|x-y| \geq r$ we take from [5] (see also [9]) that $G_{\Omega}(x, y) \geq$ $-c_{8}|x-y|^{-2} d(x)^{2} d(y)^{2}$ so that

$$
\begin{gathered}
G_{\Omega}(x, y)+2 c_{8}|x-y|^{-2} d(x)^{2} d(y)^{2} \geq c_{8}|x-y|^{-2} d(x)^{2} d(y)^{2} \\
\geq c_{8} d(x) d(y) \min \left\{1, \frac{d(x) d(y)}{|x-y|^{2}}\right\} .
\end{gathered}
$$

Since $|x-y|^{-2} \leq r^{-2}$ we end up with

$$
G_{\Omega}(x, y)+c_{9} d(x)^{2} d(y)^{2} \geq c_{8} d(x) d(y) \min \left\{1, \frac{d(x) d(y)}{|x-y|^{2}}\right\}
$$

and positive constants $c_{8}, c_{9}>0$ in this case. The proof of Theorem 1 is now complete also for $n=2$ using Corollary 8 ,

\section{Proof of Theorem 2}

This theorem was proved in [10] when $n \geq 3$ and in [9, Theorem 6.15] when $n=2$. The latter proof is quite involved and based on the extensive use of conformal maps and explicit Green functions in certain limaçons. Here we provide an alternate proof which uses the same techniques for $n=2$ as for $n \geq 3$.

Case I: $d(x) d(y) \leq|x-y|^{2}$. For this situation we have

$$
H_{\Omega}(x, y)= \begin{cases}|x-y|^{-n} d(x)^{2} d(y)^{2} & \text { if } n \neq 4, \\ \log \left(1+\frac{d(x)^{2} d(y)^{2}}{|x-y|^{4}}\right) & \text { if } n=4 .\end{cases}
$$

Then there is $c>0$ such that we find $c_{2}^{-1} H_{\Omega}(x, y) \geq c_{1} d(x)^{2} d(y)^{2}$ for $|x-y|<c$.

Case II: $d(x) d(y)>|x-y|^{2}$. Now we have

$$
H_{\Omega}(x, y)=\left\{\begin{array}{lr}
|x-y|^{4-n} & \text { if } n>4, \\
\log \left(1+\frac{d(x)^{2} d(y)^{2}}{|x-y|^{4}}\right) & \text { if } n=4, \\
d(x)^{2-n / 2} d(y)^{2-n / 2} & \text { if } n=2,3 .
\end{array}\right.
$$


Since $d(x) d(y)$ is bounded on $\Omega$ one finds for $n \geq 4$ the existence of $c>0$ such that $c_{2}^{-1} H_{\Omega}(x, y) \geq c_{1} d(x)^{2} d(y)^{2}$ for $|x-y|<c$. For dimension $n=3$ the argument is more subtle. We fix $\delta_{n}$ as in Proposition 3. Taking $d(x) d(y)<\varepsilon^{2} \frac{1+\delta_{n}}{2}$ for $\varepsilon>0$ but sufficiently small, we find that $c_{2}^{-1} H_{\Omega}(x, y) \geq c_{1} d(x)^{2} d(y)^{2}$. It remains to consider $d(x) d(y) \geq \frac{1+\delta_{n}}{2} \varepsilon^{2}$. Assume first that $|x-y|<\frac{\delta_{n}}{2} \varepsilon$ and $d(x)<\frac{1}{2} \varepsilon$. Then $d(x) d(y)<\frac{1+\delta_{n}}{2} \varepsilon^{2}$, and we are in the situation just considered. So we are left with $|x-y|<\frac{\delta_{n}}{2} \varepsilon$ and $d(x) \geq \frac{1}{2} \varepsilon$. Then we may apply Theorem 1 of [12] (see also Proposition (3) to find that for $|x-y|<\frac{1}{2} \delta_{n} \varepsilon \leq \delta_{n} \max (d(x), d(y))$ it follows that $G_{\Omega}(x, y)>0$. For dimension $n=2$ the result follows directly from Corollary 8

\section{REFERENCES}

[1] S. Agmon, A. Douglis, L. Nirenberg, Estimates near the boundary for solutions of elliptic partial differential equations satisfying general boundary conditions. I, Commun. Pure Appl. Math. 12, 623-727 (1959). MR0125307(23:A2610)

[2] T. Boggio, Sulle funzioni di Green d'ordine $m$, Rend. Circ. Mat. Palermo 20, 97-135 (1905).

[3] Kai Lai Chung, Zhong Xin Zhao, From Brownian motion to Schrödinger's equation, SpringerVerlag, Berlin, 1995. MR1329992 (96f:60140)

[4] A. Dall'Acqua, Ch. Meister, G. Sweers, Separating positivity and regularity for fourth order Dirichlet problems in 2d-domains, Analysis 25, 205-261 (2005). MR2232852 (2007b:35091)

[5] A. Dall'Acqua, G. Sweers, Estimates for Green function and Poisson kernels of higher order Dirichlet boundary value problems, J. Differential Equations 205, 466-487 (2004). MR2092867 (2005i:35065)

[6] A. Dall'Acqua, G. Sweers, On domains for which the clamped plate system is positivity preserving. In: Partial differential equations and inverse problems, pp. 133-144, Contemp. Math. 362, Amer. Math. Soc., Providence, RI, 2004. MR2091495 (2005i:35064)

[7] A. Dall'Acqua, G. Sweers, The clamped-plate equation for the limaçon. Ann. Mat. Pura Appl. (4) 184, 361-374 (2005). MR2164263(2006i:35066)

[8] R.J. Duffin, Continuation of biharmonic functions by reflection, Duke Math. J. 22, 313-324 (1955). MR.0079105 (18:29e)

[9] F. Gazzola, H.-Ch. Grunau, G. Sweers, Polyharmonic boundary value problems, Positivity preserving and nonlinear higher order elliptic equations in bounded domains. Springer Lecture Notes in Mathematics 1991, Springer-Verlag, Berlin, 2010.

[10] H.-Ch. Grunau, F. Robert, Positivity and almost positivity of biharmonic Green's functions under Dirichlet boundary conditions, Arch. Rational Mech. Anal. 195, 865-898 (2010). MR2591975

[11] H.-Ch. Grunau, G. Sweers, Positivity for perturbations of polyharmonic operators with Dirichlet boundary conditions in two dimensions, Math. Nachr. 179, 89-102 (1996). MR.1389451 (97f:35040)

[12] H.-Ch. Grunau, G. Sweers, Regions of positivity for polyharmonic Green functions in arbitrary domains, Proc. Amer. Math. Soc. 135, 3537-3546 (2007). MR2336568 (2008k:35005)

[13] J. Hadamard, Sur certains cas intéressants du problème biharmonique, Atti IV Congr. Intern. Mat. Roma 1908, (2) 12-14. Reprinted in CEuvres de Jacques Hadamard, Tome III, 1297-1299. CNRS, Paris (1968). MR0230598 (37:6158)

[14] A. Huber, On the reflection principle for polyharmonic functions, Commun. Pure Appl. Math. 9, 471-478 (1956). MR0085355(19:26e)

[15] Ju.P. Krasovskiř, Investigation of potentials connected with boundary value problems for elliptic equations (Russian), Izv. Akad. Nauk SSSR Ser. Mat. 31, 587-640 (1967). English translation in Math. USSR, Izv. 1, 569-622 (1967). MR0213727(35:4585)

[16] Ju.P. Krasovskiı̌, Isolation of singularities of the Green's function (Russian), Izv. Akad. Nauk SSSR Ser. Mat. 31, 977-1010 (1967). English translation in Math. USSR, Izv. 1, 935-966 (1967). MR0223740 (36:6788)

[17] Z. Nehari, On the biharmonic Green's function, in Studies in Mathematics and Mechanics, presented to Richard von Mises, 111-117, Academic Press, New York, 1954. MR.0064992 (16:368f) 
Fakultät für Mathematik, Оtto-von-Guericke-Universität, Postfach 4120, 39016 Magdeburg, Germany

E-mail address: hans-christoph.grunau@ovgu.de

Institut Élie Cartan, Université Henri Poincaré Nancy 1, BP 70239, 54506 Vandeuvre-Lès-Nancy Cedex, France

E-mail address: Frederic.Robert@iecn.u-nancy.fr

Mathematisches Institut, Universität Zu Köln, Weyertal 86-90, 50931 KÖln, Germany

E-mail address: gsweers@math.uni-koeln.de 